\title{
The Optimal Limit Prices of Limit Orders under an Extended Geometric Brownian Motion with Bankruptcy Risk
}

\author{
Yu-Sheng Hsu ${ }^{1}{ }^{(1)}$, Pei-Chun Chen ${ }^{2}$ and Cheng-Hsun $\mathrm{Wu}^{3, *}$ \\ 1 Department of Mathematics, National Central University, Chung-Li 320317, Taiwan; hsu@math.ncu.edu.tw \\ 2 Department of Data Science and Big Data Analytics, Providence University, Taichung City 433303, Taiwan; \\ pcchen3@pu.edu.tw \\ 3 Department of Financial Engineering and Actuarial Mathematics, Soochow University, \\ Taipei City 100006, Taiwan \\ * Correspondence: chsunwu@scu.edu.tw
}

Citation: Hsu, Y.-S.; Chen, P.-C.; Wu, C.-H. The Optimal Limit Prices of Limit Orders under an Extended Geometric Brownian Motion with Bankruptcy Risk. Mathematics 2021, 9, 54. https://doi.org/10.3390/ math 9010054

Received: 4 December 2020 Accepted: 25 December 2020 Published: 29 December 2020

Publisher's Note: MDPI stays neutral with regard to jurisdictional clai$\mathrm{ms}$ in published maps and institutional affiliations.

Copyright: $\odot 2020$ by the authors. Licensee MDPI, Basel, Switzerland. This article is an open access article distributed under the terms and conditions of the Creative Commons Attribution (CC BY) license (https:// creativecommons.org/licenses/by/ $4.0 /)$.

\begin{abstract}
In the Black and Scholes system, the underlying asset price model follows geometric Brownian motion (GBM) with no bankruptcy risk. While GBM is a commonly used model in financial markets, bankruptcy risk should be considered in the case of a severe economic crisis, such as that caused by the COVID-19 pandemic. The omission of bankruptcy risk could considerably influence the setting of a trading strategy. In this article, we adopt an extended GBM model that considers the bankruptcy risk and study its optimal limit price problem. A limit order is a classical trading strategy for investing in stocks. First, we construct the explicit expressions of the expected discounted profit functions for sell and buy limit orders and then derive their optimal limit prices. Furthermore, via sensitivity analysis, we discuss the influence of the omission of bankruptcy risk in executing limit orders.
\end{abstract}

Keywords: geometric Brownian motion; Black-Scholes model; limit orders; optimal limit prices

\section{Introduction}

Limit orders are a popular and typical mode for trading stocks. If an investor has a long position on one stock, she/he can set a sell limit order with a specific selling price in order to obtain a profit. Alternatively, if one has a short position on a security, she/he can set a buy limit order with a specific buying price to obtain a profit. Hence, a reasonable execution price is a crucial characteristic for limit orders.

This problem is a popular and worthy issue in finance and can be used to investigate optimal investment timing. It has been well solved for the case of geometric Brownian motion. McDonald and Siegel [1] first studied this problem based on geometric Brownian motion and provided the explicit solution via the profit criterion. There are many other criteria for setting the optimal timing. Shiryaev et al. [2] adopted minimizing the expected relative error as a criterion to study the optimal selling timing. Dai et al. [3] utilize the squared error between the selling price and the global maximum price to study the optimal selling strategy. From an empirical point of view, Handa and Schwartz [4] discussed the effect of limit order trading on the market. Lo et al. [5] applied survival analysis to historical limit order data to develop a strategy for setting the execution time of limit orders and empirically showed that adopting geometric Brownian motion with the first passage time setting had poor performance in limit order execution. Agliardi [6] and Agliardi and Gencay [7] constructed stochastic dynamic programming problems for limit order execution based on the limit order book and provided their explicit solutions. In this article, in order to discuss the effect of the omission of bankruptcy risk and analyze the expected discounted profits directly, we adopt the profit criterion with the first passage time setting to derive the optimal limit price. Furthermore, we compare the performance of the stock price model with bankruptcy risk with that of the model with no bankruptcy risk, which is 
that with geometric Brownian motion, via optimal limit prices and expected discounted profits.

Owing to the positive behavior, geometric Brownian motion fails to illustrate the real occurrence of bankruptcy. Ignoring the occurrence of bankruptcies could cause geometric Brownian motion to provide higher estimations of the future stock price. Under the global economic crisis, both economics and finance are severely shocked. A firm will encounter troublesome difficulties in running its business and then the bankruptcy risk will increase. Especially when studying the impacts of the COVID-19 pandemic, bankruptcy risk should be integrated into the stock price model. Several articles about the impact of the COVID-19 pandemic on economics and finance can be found in Goodell [8], Baldwin and Weder di Mauro [9], and Zhang et al. [10].

Therefore, we consider the stock price model, which is proposed by Hsu and Wu [11], as follows:

$$
S_{b}(t)=\left(c_{1} e^{a B(t)+b t}-c_{2} e^{-a B(t)-b t}\right) I_{\left\{t<T_{0}\right\}}=Y(t) I_{\left\{t<T_{0}\right\}},
$$

where $Y(t)=c_{1} e^{a B(t)+b t}-c_{2} e^{-a B(t)-b t}, c_{1} \geq c_{2}>0, a>0, b \in \mathbb{R}$ and $T_{0}=\inf \{t \mid Y(t)=0\}$. Based on $\mathrm{Hsu}$ and $\mathrm{Wu}$ [11], model (1) can extend geometric Brownian motion to illustrate bankruptcy risk and reduce its overestimation of prices. Note that there exists an equivalent martingale measure for model (1) and then model (1) is an arbitrage-free market model with admissible strategies, see Hsu and Wu [11] for more details. Intuitively, a higher estimated price could cause an investor to set a higher limit price for a sell limit order and have more risk. For a buy limit order, if an investor adopts a model that overestimates the price, she/he could set a higher executed price and lose profits. Thus, we discuss the optimal limit price for a limit order based on model (1) and compare its performance with that of geometric Brownian motion in order to explore the effect of the omission of bankruptcy risk in trading stocks via limit orders.

The remainder of this article is organized as follows. We introduce the strategy of setting the limit price and construct the expression of the expected discounted profits in Section 2. The existence of optimal limit prices is verified in Section 3. The results on setting the strategies for limit orders at any time are presented in Section 4 . The influence of the omission of bankruptcy risk is discussed using sensitivity analysis in Section 5 . The conclusions are given in Section 6.

\section{The Expected Discounted Profit Function for Limit Prices}

Assume that the stock price follows model (1). In this section, we derive the expected discounted profit function and construct the criterion for setting the limit price. We adopt the profit criterion that was proposed in McDonald and Siegel [1]. Note that the optimal investment timing problem based on the profit criterion with constant investment costs is set using the first passage time setting, see Reference [1] for more details. Furthermore, the superiority of the first passage time setting with the profit criterion is that the explicit expression of the expected discounted profit function can be derived and then investors can tractably analyze the profits of their investments.

For a sell limit order, we assume that the cost of possessing a stock at the beginning is $L \geq S_{b}(0)=c_{1}-c_{2}$. We explore the optimal execution timing based on the first passage time setting and denote the first passage time as $T_{x}=\inf \left\{t \mid S_{b}(t)=x\right\}$ with the specific limit price $x$. Assume that the sum of trading fee and tax is proportional to the stock price with a constant rate $\sigma$, where $0<\sigma<1$. Hence, we construct the discounted profit of a sell limit order as:

$$
e^{-r\left(T_{x} \wedge T_{0}\right)}\left[(1-\sigma) S_{b}\left(T_{x}\right)-L\right]=(1-\sigma) e^{-r\left(T_{x} \wedge T_{0}\right)}\left[S_{b}\left(T_{x}\right)-L_{\sigma}^{-}\right],
$$

where $r$ is the constant interest rate, $L_{\sigma}^{-}=\frac{L}{1-\sigma}$ and $T_{0}$ represents the bankruptcy time. Then, the discounted profit of a sell limit order can be rewritten as:

$$
(1-\sigma)\left[\left(x-L_{\sigma}^{-}\right) I_{\left\{T_{x}<T_{0}\right\}} e^{-r T_{x}}-L_{\sigma}^{-} I_{\left\{T_{0}<T_{x}\right\}} e^{-r T_{0}}\right] .
$$


We construct the expected discounted profit function of a sell limit order as:

$$
\begin{aligned}
\mathrm{g}_{\mathrm{s}}(x) & =(1-\sigma) E\left[e^{-r\left(T_{x} \wedge T_{0}\right)}\left(S_{b}\left(T_{x} \wedge T_{0}\right)-L_{\sigma}^{-}\right)\right] \\
& =(1-\sigma)\left[\left(x-L_{\sigma}^{-}\right) E\left(I_{\left\{T_{x}<T_{0}\right\}} e^{-r T_{x}}\right)-L_{\sigma}^{-} E\left(I_{\left\{T_{0}<T_{x}\right\}} e^{-r T_{0}}\right)\right],
\end{aligned}
$$

for $x \geq L_{\sigma}^{-}$. The positive term in (2) represents the expected profits when the stock price hits $x$ before the bankruptcy time, and the minus term in (2) represents the loss when bankruptcy occurs before $T_{x}$. In the following proposition, we derive the exact expression of the expected discounted profit function $\mathrm{g}_{\mathrm{s}}(x)$.

Proposition 1. Assume the cost of possessing the underlying asset at the beginning is $L \geq S_{b}(0)=$ $c_{1}-c_{2}$ and the limit price $x \geq L_{\sigma}^{-}$. The expected discounted profit function of a sell limit order is:

$$
\mathrm{g}_{\mathrm{s}}(x)=(1-\sigma)\left[\frac{\left(x-L_{\sigma}^{-}\right) e^{\mu D(x)} \sinh \left(-\mu_{r} D(0)\right)-L_{\sigma}^{-} e^{\mu D(0)} \sinh \left(\mu_{r} D(x)\right)}{\sinh \left(\mu_{r}(D(x)-D(0))\right)}\right],
$$

for $x \geq L_{\sigma}^{-}$, where $\mu=\frac{b}{a}, \mu_{r}=\sqrt{2 r+\mu^{2}}$ and $D(y)=\frac{1}{a} \ln \left(\frac{y+\sqrt{y^{2}+4 c_{1} c_{2}}}{2 c_{1}}\right)$.

Proof. First, we demonstrate the expressions of $E\left(I_{\left\{T_{w}^{B}<T_{v}^{B}\right\}} e^{-r T_{w}^{B}}\right)$ and $E\left(I_{\left\{T_{v}^{B}<T_{w}^{B}\right\}} e^{-r T_{v}^{B}}\right)$, where $v<0<w$ and $T_{y}^{B}=\inf \{t \mid B(t)+\mu t=y\}$. Let $Z(t)=\exp \left(-\mu B(t)-\frac{1}{2} \mu^{2} t\right)$ and $W(t)=B(t)+\mu t$. By Girsanov's theorem and the optional sampling theorem, we obtain

$$
\begin{aligned}
& E\left(I_{\left\{T_{w}^{B}<T_{v}^{B}<t\right\}} e^{-r T_{w}^{B}}\right)=E\left(I_{\left\{T_{w}^{B}<T_{v}^{B}<t\right\}} e^{-r\left(T_{w}^{B} \wedge T_{v}^{B} \wedge t\right)}\right) \\
& =E^{P}\left(I_{\left\{T_{w}^{B}<T_{v}^{B}<t\right\}} e^{-r\left(T_{w}^{B} \wedge T_{v}^{B} \wedge t\right)} Z^{-1}(t)\right) \\
& =E^{P}\left(E^{P}\left(I_{\left\{T_{w}^{B}<T_{v}^{B}<t\right\}} e^{-r\left(T_{w}^{B} \wedge T_{v}^{B} \wedge t\right)} Z^{-1}(t) \mid \mathcal{F}_{T_{w}^{B} \wedge T_{v}^{B} \wedge t}\right)\right) \\
& =E^{P}\left(I_{\left\{T_{w}^{B}<T_{v}^{B}<t\right\}} e^{-r\left(T_{w}^{B} \wedge T_{v}^{B} \wedge t\right)} Z^{-1}\left(T_{w}^{B} \wedge T_{v}^{B} \wedge t\right)\right) \\
& =E^{P}\left(I_{\left\{T_{w}^{B}<T_{v}^{B}<t\right\}} e^{-r\left(T_{w}^{B} \wedge T_{v}^{B} \wedge t\right)} e^{\mu W\left(T_{w}^{B} \wedge T_{v}^{B} \wedge t\right)-\frac{1}{2} \mu^{2}\left(T_{w}^{B} \wedge T_{v}^{B} \wedge t\right)}\right) \\
& =E^{P}\left(I_{\left\{T_{w}^{B}<T_{v}^{B}<t\right\}} e^{-r T_{w}^{B}} e^{\mu w-\frac{1}{2} \mu^{2} T_{w}^{B}}\right) \\
& =e^{\mu w} E^{P}\left(I_{\left\{T_{w}^{B}<T_{v}^{B}<t\right\}} e^{-\left(r+\frac{1}{2} \mu^{2}\right) T_{w}^{B}}\right),
\end{aligned}
$$

where $P$ is the probability measure derived by Girsanov's theorem such that the Brownian motion with drift, $\{B(t)+\mu t\}$, is a standard Brownian motion under $P$. It is clear that:

$$
I_{\left\{T_{w}^{B}<T_{v}^{B}<t\right\}} e^{-k T_{w}^{B}} \rightarrow I_{\left\{T_{w}^{B}<T_{v}^{B}\right\}} e^{-k T_{w}^{B}}
$$

for any positive constant $k$ as $t$ approaches infinity and:

$$
0<I_{\left\{T_{w}^{B}<T_{v}^{B}<t\right\}} e^{-k T_{w}^{B}} \leq 1 .
$$

By the bounded convergence theorem, we obtain:

$$
E\left(I_{\left\{T_{w}^{B}<T_{v}^{B}<t\right\}} e^{-r T_{w}^{B}}\right) \rightarrow e^{\mu w} E^{P}\left(I_{\left\{T_{w}^{B}<T_{v}^{B}\right\}} e^{-\left(r+\frac{1}{2} \mu^{2}\right) T_{w}^{B}}\right)
$$

as $t \rightarrow \infty$. Hence, we have:

$$
\begin{aligned}
E\left(I_{\left\{T_{w}^{B}<T_{v}^{B}\right\}} e^{-r T_{w}^{B}}\right) & =e^{\mu w} E^{P}\left(I_{\left\{T_{w}^{B}<T_{v}^{B}\right\}} e^{-\left(r+\frac{1}{2} \mu^{2}\right) T_{w}^{B}}\right) \\
& =e^{\mu w} \frac{\sinh \left(-v \sqrt{2 r+\mu^{2}}\right)}{\sinh \left((w-v) \sqrt{2 r+\mu^{2}}\right)} .
\end{aligned}
$$


Similarly, we have:

$$
\begin{aligned}
& E\left(I_{\left\{T_{v}^{B}<T_{w}^{B}\right\}} e^{-r T_{v}^{B}}\right)=e^{\mu v} E^{P}\left(I_{\left\{T_{v}^{B}<T_{w}^{B}\right\}} e^{-\left(r+\frac{1}{2} \mu^{2}\right) T_{v}^{B}}\right) \\
& =e^{\mu v} \frac{\sinh \left(w \sqrt{2 r+\mu^{2}}\right)}{\sinh \left((w-v) \sqrt{2 r+\mu^{2}}\right)} \text {. }
\end{aligned}
$$

Expressions (5) and (6) can be derived directly, see Karatzas and Shreve [12], p. 100 for more details. Note that based on (5) and (6), for $x \geq L_{\sigma}^{-}$, the expected discounted profit function of the sell limit order is:

$$
\mathrm{g}_{\mathbf{s}}(x)=(1-\sigma)\left[\frac{\left(x-L_{\sigma}^{-}\right) e^{\mu D(x)} \sinh \left(-\mu_{r} D(0)\right)-L_{\sigma}^{-} e^{\mu D(0)} \sinh \left(\mu_{r} D(x)\right)}{\sinh \left(\mu_{r}(D(x)-D(0))\right)}\right],
$$

where $\mu=\frac{b}{a}, \mu_{r}=\sqrt{2 r+\mu^{2}}$ and $D(y)=\frac{1}{a} \ln \left(\frac{y+\sqrt{y^{2}+4 c_{1} c_{2}}}{2 c_{1}}\right)$.

Next, we derive the expected profit function for the buy limit order. Assume that an investor acquires income $L \leq S_{b}(0)=c_{1}-c_{2}$ from a short position at the beginning and the costs of short covering are $(1+\sigma) S_{b}\left(T_{x}\right)$. Then, the discounted profit of a limit order follows:

$$
e^{-r T_{x}}\left(L-(1+\sigma) S_{b}\left(T_{x}\right)\right)=(1+\sigma) e^{-r T_{x}}\left(L_{\sigma}^{+}-S_{b}\left(T_{x}\right)\right),
$$

where $x$ is the limit price of the buy limit order, $L_{\sigma}^{+}=\frac{L}{1+\sigma}$ and $0<x \leq L_{\sigma}^{+}$. Since $T_{x} \wedge T_{0}=T_{x}$, we obtain the expected discounted profit function of the buy limit order as:

$$
\mathrm{g}_{\mathrm{b}}(x)=(1+\sigma)\left(L_{\sigma}^{+}-x\right) E\left(e^{-r T_{x}}\right),
$$

for $0 \leq x \leq L_{\sigma}^{+}$. Since $T_{x}=\inf \{t \mid B(t)+\mu t=D(x)\}$, we obtain:

$$
\mathrm{g}_{\mathrm{b}}(x)=(1+\sigma)\left(L_{\sigma}^{+}-x\right)\left(\frac{x+\sqrt{x^{2}+4 c_{1} c_{2}}}{2 c_{1}}\right)^{\frac{1}{a}\left(\mu+\sqrt{\mu^{2}+2 r}\right)},
$$

based on the Laplace transform of the first passage time of Brownian motion with drift.

We aim to construct the optimal sell/buy limit prices based on the following criterion:

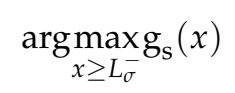

and:

$$
\arg \max _{0 \leq x \leq L_{\sigma}^{+}} \mathrm{g}_{\mathrm{b}}(x)
$$

respectively. In the next subsection, we clarify the existence of the optimal sell limit price and derive the expression of the optimal buy limit price.

\section{The Optimal Limit Prices}

In this section, we discuss the optimal limit price for sell and buy limit orders. In the following theorem, we verify the existence of the optimal limit price for a sell limit order and confirm that the optimal sell limit price can be found in some bounded and closed interval.

Theorem 1. If $r>|b|+\frac{a^{2}}{2}, \mathrm{~g}_{\mathrm{s}}(x)$ attains its absolute maximum value on $\left[L_{\sigma}^{-}, \infty\right)$. Moreover, there is a number $\delta>L_{\sigma}^{-}$such that:

$$
\operatorname{maxg}_{x \geq L_{\bar{\sigma}}^{-}} \mathrm{g}_{\mathrm{s}}(x)=\max _{x \in\left[L_{\sigma}^{-}, \delta\right]} \mathrm{g}_{\mathrm{s}}(x)
$$


Proof. First, we claim that $\mathrm{g}_{\mathrm{s}}(x)$ converges to a constant as $x$ tends to infinity. Then, we show that $\mathrm{g}_{\mathrm{s}}(x)$ attains its absolute maximum value on some closed and bounded interval. Let $y=x+\sqrt{x^{2}+4 c_{1} c_{2}}$ and $z=\frac{\mu_{r}}{a}$. Define $g_{1}(y)=\mathrm{g}_{\mathrm{s}}(x)$, and then we have:

$$
\mathrm{g}_{1}(y)=(1-\sigma) \frac{K_{1}\left(y^{2}-2 L_{\sigma}^{-} y-4 c_{1} c_{2}\right) y^{\frac{\mu}{a}+z}-L_{\sigma}^{-} e^{\mu D(0)}\left(\left(2 c_{1}\right)^{-z} y^{2 z+1}-\left(2 c_{1}\right)^{z} y\right)}{e^{-\mu_{r} D(0)}\left(2 c_{1}\right)^{-z} y^{2 z+1}-e^{\mu_{r} D(0)}\left(2 c_{1}\right)^{z} y},
$$

for $y \geq L_{\sigma}^{-}+\sqrt{\left(L_{\sigma}^{-}\right)^{2}+4 c_{1} c_{2}}$, where $K_{1}=\left(2 c_{1}\right)^{-\frac{\mu}{a}} \sinh \left(-\mu_{r} D(0)\right)$. Since $r>|b|+\frac{a^{2}}{2}$, we have $z-\frac{\mu}{a}-1>0$, and then $2 z+1>z+\frac{\mu}{a}+2$. Hence, we obtain:

$$
\mathrm{g}_{1}(y) \rightarrow-(1-\sigma) L_{\sigma}^{-} e^{D(0)\left(\mu+\sqrt{2 r+\mu^{2}}\right)}
$$

as $y \rightarrow \infty$. This implies $\mathrm{g}_{\mathrm{s}}(x) \rightarrow-(1-\sigma) L_{\sigma}^{-} e^{D(0)\left(\mu+\sqrt{2 r+\mu^{2}}\right)}$ as $x \rightarrow \infty$. Since $D\left(L_{\sigma}^{-}\right)>0$ and $D\left(L_{\sigma}^{-}\right)-D(0)>0$, we have $\sinh \left(\mu_{r}\left(D\left(L_{\sigma}^{-}\right)-D(0)\right)\right)>0$ and $\sinh \left(\mu_{r} D\left(L_{\sigma}^{-}\right)\right)>0$. Furthermore, owing to $D\left(L_{\sigma}^{-}\right)-D(0)>D\left(L_{\sigma}^{-}\right)$, we have:

$$
0<\frac{\sinh \left(\mu_{r} D\left(L_{\sigma}^{-}\right)\right)}{\sinh \left(\mu_{r}\left(D\left(L_{\sigma}^{-}\right)-D(0)\right)\right)}<1
$$

and then we obtain:

$$
\mathrm{g}_{\mathrm{s}}\left(L_{\sigma}^{-}\right)=(1-\sigma) \frac{-L_{\sigma}^{-} e^{\mu D(0)} \sinh \left(\mu_{r} D\left(L_{\sigma}^{-}\right)\right)}{\sinh \left(\mu_{r}\left(D\left(L_{\sigma}^{-}\right)-D(0)\right)\right)}>-(1-\sigma) L_{\sigma}^{-} e^{D(0)\left(\mu+\sqrt{2 r+\mu^{2}}\right)} .
$$

Choose $\epsilon>0$ such that $\mathrm{g}_{\mathrm{s}}\left(L_{\sigma}^{-}\right)>\epsilon-(1-\sigma) L_{\sigma}^{-} e^{D(0)\left(\mu+\sqrt{2 r+\mu^{2}}\right)}$. Then, there exists a number $\delta>0$ such that:

$$
-\epsilon-(1-\sigma) L_{\sigma}^{-} e^{D(0)\left(\mu+\sqrt{2 r+\mu^{2}}\right)}<\mathrm{g}_{\mathrm{s}}(x)<\epsilon-(1-\sigma) L_{\sigma}^{-} e^{D(0)\left(\mu+\sqrt{2 r+\mu^{2}}\right)}
$$

for $x \in[\delta, \infty)$. Consequently, we obtain $\max _{x \geq L_{\sigma}^{-}} \mathrm{g}_{\mathrm{s}}(x)=\max _{x \in\left[L_{\sigma}^{-}, \delta\right]} \mathrm{g}_{\mathrm{s}}(x)$. Since $\mathrm{g}_{\mathrm{s}}(x)$ is continuous on $\left[L_{\sigma}^{-}, \delta\right]$, we verify that $\mathrm{g}_{\mathrm{s}}(x)$ attains its absolute maximum value on $\left[L_{\sigma}^{-}, \infty\right)$ by the extreme value theorem.

Owing to (4) being so complex that it is difficult to obtain the explicit form of the optimal sell limit price, we can calculate this optimal price via a numerical method directly based on Theorem 1 .

We proceed to derive the explicit form of the optimal limit price for the buy limit order in the following theorem.

Theorem 2. Let $z_{1}=\frac{1}{a}\left(\mu+\sqrt{\mu^{2}+2 r}\right)$ and $x_{b}^{*}=\frac{L_{\sigma}^{+} z_{1}^{2}-\sqrt{z_{1}^{2}\left(L_{\sigma}^{+}\right)^{2}+4 c_{1} c_{2}\left(z_{1}^{2}-1\right)}}{z_{1}^{2}-1}$. We have the following conclusions.

(a) Assume $z_{1}^{2}>1$. If $\frac{2 \sqrt{c_{1} c_{2}}}{z_{1}} \leq L_{\sigma}^{+} \leq c_{1}-c_{2}$, then $\max _{x \in\left[0, L_{\sigma}^{+}\right]} \mathrm{g}_{\mathrm{b}}(x)=\mathrm{g}_{\mathrm{b}}\left(x_{b}^{*}\right)$. If $0<L<$ $\min \left(\frac{2 \sqrt{c_{1} c_{2}}}{z_{1}}, c_{1}-c_{2}\right)$, then $\max _{x \in\left[0, L_{\sigma}^{+}\right]} \mathrm{g}_{\mathrm{b}}(x)=\mathrm{g}_{\mathrm{b}}(0)$.

(b) Assume $0<z_{1}^{2}<1$ and $L_{\sigma}^{+}>2 \sqrt{c_{1} c_{2}\left(z_{1}^{-2}-1\right)}$. If $\frac{2 \sqrt{c_{1} c_{2}}}{z_{1}} \leq L_{\sigma}^{+} \leq c_{1}-c_{2}$, then $\max _{x \in\left[0, L_{\sigma}^{+}\right]} \mathrm{g}_{\mathrm{b}}(x)=\mathrm{g}_{\mathrm{b}}\left(x_{b}^{*}\right)$. If $0<L_{\sigma}^{+}<\min \left(\frac{2 \sqrt{c_{1} c_{2}}}{z_{1}}, c_{1}-c_{2}\right)$, then $\max _{x \in\left[0, L_{\sigma}^{+}\right]} \mathrm{g}_{\mathrm{b}}(x)=\mathrm{g}_{\mathrm{b}}(0)$. 
Proof. It is clear that:

$$
\mathrm{g}_{\mathrm{b}}^{\prime}(x)=(1+\sigma) \frac{z_{1}\left(L_{\sigma}^{+}-x\right)-\sqrt{x^{2}+4 c_{1} c_{2}}}{\sqrt{x^{2}+4 c_{1} c_{2}}}\left(\frac{x+\sqrt{x^{2}+4 c_{1} c_{2}}}{2 c_{1}}\right)^{z_{1}} .
$$

Based on the following properties:

(i) If $z_{1}^{2}>1$, then $\frac{L_{\sigma}^{+} z_{1}^{2}+\sqrt{z_{1}^{2}\left(L_{\sigma}^{+}\right)^{2}+4 c_{1} c_{2}\left(z_{1}^{2}-1\right)}}{z_{1}^{2}-1}>L_{\sigma}^{+}$, and

(ii) If $z_{1}^{2}<1$ and $L_{\sigma}^{+} \geq 2 \sqrt{c_{1} c_{2}\left(z_{1}^{-2}-1\right)}$, then $\frac{L_{\sigma}^{+} z_{1}^{2}+\sqrt{z_{1}^{2}\left(L_{\sigma}^{+}\right)^{2}+4 c_{1} c_{2}\left(z_{1}^{2}-1\right)}}{z_{1}^{2}-1}<0$, we obtain that:

$$
x_{b}^{*}=\frac{L_{\sigma}^{+} z_{1}^{2}-\sqrt{z_{1}^{2}\left(L_{\sigma}^{+}\right)^{2}+4 c_{1} c_{2}\left(z_{1}^{2}-1\right)}}{z_{1}^{2}-1}
$$

is the only candidate critical number for $\mathrm{g}_{\mathrm{b}}(x)$ on $\left[0, L_{\sigma}^{+}\right]$. Note that we need $L_{\sigma}^{+} \geq$ $2 \sqrt{c_{1} c_{2}\left(z_{1}^{-2}-1\right)}$ to ensure that $\sqrt{z_{1}^{2}\left(L_{\sigma}^{+}\right)^{2}+4 c_{1} c_{2}\left(z_{1}^{2}-1\right)}$ is defined when $z_{1}^{2}<1$. Since:

We proceed to prove (a). Assume $z_{1}^{2}>1$. If $\frac{2 \sqrt{c_{1} c_{2}}}{z_{1}} \leq L_{\sigma}^{+} \leq c_{1}-c_{2}$, then $0 \leq x_{b}^{*} \leq L_{\sigma}^{+}$.

$$
\mathrm{g}_{\mathrm{b}}^{\prime}(x)\left\{\begin{array}{l}
\geq 0, \quad \text { if } x \in\left[0, x_{b}^{*}\right] \\
\leq 0, \quad \text { if } x \in\left[x_{b}^{*}, L_{\sigma}^{+}\right]
\end{array}\right.
$$

we have $\max _{x \in\left[0, L_{\sigma}^{+}\right]} \mathrm{g}_{\mathrm{b}}(x)=\mathrm{g}_{\mathrm{b}}\left(x_{b}^{*}\right)$. If $0<L_{\sigma}^{+}<\min \left(\frac{2 \sqrt{c_{1} c_{2}}}{z_{1}}, c_{1}-c_{2}\right)$, then $x_{b}^{*}<0$ and $\max _{x \in\left[0, L^{+}\right]} \mathrm{g}_{\mathrm{b}}(x)=\mathrm{g}_{\mathrm{b}}(0)$. The conclusion in (b) follows easily by similar arguments applied in (a).

\section{The Results for Entering a Long or Short Position at Any Time}

To set a more elastic limit order, we assume that the investor can enter a long or short position at any time $t_{1}$ with the stock price $S_{b}\left(t_{1}\right)=y$. In this section, we construct the expected discounted profit functions and their optimal limit prices for the limit orders in which the investor enters a position at any time $t_{1}$ with the stock price $S_{b}\left(t_{1}\right)=y$.

First, we demonstrate the results for the sell limit order in which the investor has bought the stock at time $t_{1}$ with price $S_{b}\left(t_{1}\right)=y$. Define the conditional stock price $\left(S_{b}(t) \mid S_{b}\left(t_{1}\right)=y\right)$ at time $t$, given $S_{b}\left(t_{1}\right)=y$, and its first passage time:

$$
T_{k}^{C}=\inf \left\{t \mid\left(S_{b}(t) \mid S_{b}\left(t_{1}\right)=y\right)=k\right\} .
$$

We define the discounted profit of the sell limit order, given $S_{b}\left(t_{1}\right)=y$, as:

$$
e^{-r\left(\left(T_{x}^{C} \wedge T_{0}^{C}\right)-t_{1}\right)}\left((1-\sigma)\left(S_{b}\left(T_{x}^{C}\right) \mid S_{b}\left(t_{1}\right)=y\right)-L\right),
$$

for $L \geq y$, where $L$ represents the cost of entering long position at time $t_{1}$ and $x$ represent the sell limit price. Define:

$$
T_{k, 1}^{C, B}=\inf \left\{t \mid\left(B(t)+\frac{b}{a} t\right)-\left(B\left(t_{1}\right)+\frac{b}{a} t_{1}\right)=D(k)-D(y)\right\} .
$$

Since $T_{x}^{C} \wedge T_{0}^{C}=T_{x, 1}^{C, B} \wedge T_{0,1}^{C, B}$, we can rewrite (8) as:

$$
\begin{gathered}
(1-\sigma) e^{-r\left(\left(T_{x}^{C} \wedge T_{0}^{C}\right)-t_{1}\right)}\left(\left(S_{b}\left(T_{x}^{C}\right) \mid S_{b}\left(t_{1}\right)=y\right)-L_{\sigma}^{-}\right) \\
=(1-\sigma) e^{r t_{1}}\left[I_{\left\{T_{x, 1}^{C, B}<T_{0,1}^{C, B}\right\}} e^{-r T_{x, 1}^{C, B}}\left(x-L_{\sigma}^{-}\right)-L_{\sigma}^{-} I_{\left\{T_{0,1}^{C, B}<T_{x, 1}^{C, B}\right\}} e^{-r T_{0,1}^{C, B}}\right],
\end{gathered}
$$


where $L_{\sigma}^{-}=\frac{L}{1-\sigma}$. Then, we obtain the expected discounted profit function of the sell limit order, given $S_{b}\left(t_{1}\right)=y$, as follows:

$$
\begin{aligned}
& \left(\mathrm{g}_{\mathrm{s}} \mid S_{b}\left(t_{1}\right)=y\right)(x) \\
& =e^{r t_{1}}(1-\sigma)\left[\left(x-L_{\sigma}^{-}\right) E\left(I_{\left\{T_{x, 1}^{C, B}<T_{0,1}^{C, B}\right\}} e^{-r T_{x, 1}^{C, B}}\right)-L_{\sigma}^{-} E\left(I_{\left\{T_{0,1}^{C, B}<T_{x, 1}^{C, B}\right\}} e^{-r T_{0,1}^{C, B}}\right)\right],
\end{aligned}
$$

for $x \geq L_{\sigma}^{-}$. Define $T_{k, 2}^{C, B}=\inf \left\{t \mid B(t)+\frac{b}{a} t=D(k)-D(y)\right\}$. Since $\left(T_{x, 1}^{C, B}, T_{0,1}^{C, B}\right)$ and $\left(t_{1}+T_{x, 2}^{C, B}, t_{1}+T_{0,2}^{C, B}\right)$ have the same joint distribution, we can rewrite $\left(\mathrm{g}_{\mathrm{s}} \mid S_{b}\left(t_{1}\right)=y\right)(x)$ as:

$$
\begin{aligned}
& \left(\mathrm{g}_{\mathrm{s}} \mid S_{b}\left(t_{1}\right)=y\right)(x) \\
& =(1-\sigma)\left[\left(x-L_{\sigma}^{-}\right) E\left(I_{\left\{T_{x, 2}^{C, B}<T_{0,2}^{C, B}\right\}} e^{-r T_{x, 2}^{C, B}}\right)-L_{\sigma}^{-} E\left(I_{\left\{T_{0,2}^{C, B}<T_{x, 2}^{C, B}\right\}} e^{-r T_{0,2}^{C, B}}\right)\right] .
\end{aligned}
$$

By (5) and (6), we obtain the expected discounted profit function of the sell limit order, given $S_{b}\left(t_{1}\right)=y$, for $x \geq L_{\sigma}^{-}$, as follows:

$$
\begin{aligned}
& \left(\mathrm{g}_{\mathrm{s}} \mid S_{b}\left(t_{1}\right)=y\right)(x) \\
& =(1-\sigma)\left[\frac{\left(x-L_{\sigma}^{-}\right) e^{\mu D_{1}(x, y)} \sinh \left(\mu_{r} D_{1}(y, 0)\right)-L_{\sigma} e^{\mu D_{1}(0, y)} \sinh \left(\mu_{r} D_{1}(x, y)\right)}{\sinh \left(\mu_{r} D_{1}(x, 0)\right)}\right],
\end{aligned}
$$

where $D_{1}\left(k_{1}, k_{2}\right)=D\left(k_{1}\right)-D\left(k_{2}\right), \mu=\frac{b}{a}$ and $\mu_{r}=\sqrt{2 r+\mu^{2}}$. In the following theorem, we clarify the existence of the optimal limit price for a sell limit order, given $S_{b}\left(t_{1}\right)=y$ and its proof is given in Appendix A.

Theorem 3. If $r>|b|+\frac{a^{2}}{2}$, then $\left(\mathrm{g}_{\mathrm{s}} \mid S_{b}\left(t_{1}\right)=y\right)(x)$ attains its absolute maximum value on $\left[L_{\sigma}^{-}, \infty\right]$. In addition, there is a number $\delta>L$ such that:

$$
\max _{x \geq L_{\sigma}^{-}}\left(\mathrm{g}_{\mathrm{s}} \mid S_{b}\left(t_{1}\right)=y\right)(x)=\max _{x \in\left[L_{\sigma}^{-}, \delta\right]}\left(\mathrm{g}_{\mathrm{s}} \mid S_{b}\left(t_{1}\right)=y\right)(x) .
$$

Proof. See Appendix A. $\square$

We proceed to derive the results for a buy limit order given $S_{b}\left(t_{1}\right)=y$. The discounted profit of the buy limit order that enters the short position at time $t_{1}$, given $S_{b}\left(t_{1}\right)=y$, is as follows:

$$
e^{-r\left(\left(T_{x}^{C} \wedge T_{0}^{C}\right)-t_{1}\right)}\left(L-(1+\sigma)\left(S_{b}\left(T_{x}^{C}\right) \mid S_{b}\left(t_{1}\right)=y\right)\right),
$$

for $L \leq y$ and $x \geq 0$. Note that $L$ represents the income of the investor that enters a short position of the stock at time $t_{1}$. Since $T_{x}^{C} \geq T_{0}^{C}$, we have:

$$
e^{-r\left(\left(T_{x}^{C} \wedge T_{0}^{C}\right)-t_{1}\right)}\left(L-(1+\sigma)\left(S_{b}\left(T_{x}^{C}\right) \mid S_{b}\left(t_{1}\right)=y\right)\right)=(1+\sigma) e^{-r T_{x}^{C}}\left(L_{\sigma}^{+}-x\right),
$$

where $L_{\sigma}^{+}=\frac{L}{1+\sigma}$. Since $T_{x}^{C}=T_{x, 1}^{C, B}$ and $T_{x, 1}^{C, B}$ has the same distribution as $t_{1}+T_{x, 2}^{C, B}$, we obtain the expected discounted profit function as follows:

$$
\begin{aligned}
\left(\mathrm{g}_{\mathrm{b}} \mid S_{b}\left(t_{1}\right)=y\right)(x) & =(1+\sigma)\left(L_{\sigma}^{+}-x\right) E\left(e^{-r T_{x}^{C}}\right) \\
& =(1+\sigma)\left(L_{\sigma}^{+}-x\right)\left(\frac{x+\sqrt{x^{2}+4 c_{1} c_{2}}}{y+\sqrt{y^{2}+4 c_{1} c_{2}}}\right)^{\frac{1}{a}\left(\mu+\sqrt{\mu^{2}+2 r}\right)},
\end{aligned}
$$

for $L \leq y$ and $0 \leq x \leq L_{\sigma}^{+}$. We provide the explicit expression for the optimal limit price of a buy limit order, given $S_{b}\left(t_{1}\right)=y$, in the following theorem. The proof is given in the Appendix A. 
Theorem 4. Let $x_{b, C}^{*}=\frac{L_{\sigma}^{+} z_{1}^{2}-\sqrt{z_{1}^{2}\left(L_{\sigma}^{+}\right)^{2}+4 c_{1} c_{2}\left(z_{1}^{2}-1\right)}}{z_{1}^{2}-1}$. We have the following conclusions:

(a) Assume $z_{1}^{2}>1$. If $\frac{2 \sqrt{c_{1} c_{2}}}{z_{1}} \leq L_{\sigma}^{+} \leq y$, then $\max _{x \in\left[0, L_{\sigma}^{+}\right]}\left(g_{b} \mid S_{b}\left(t_{1}\right)=y\right)(x)=\left(g_{b} \mid S_{b}\left(t_{1}\right)=y\right)$ $\left(x_{b, C}^{*}\right)$. If $0<L_{\sigma}^{+}<\frac{2 \sqrt{c_{1} c_{2}}}{z_{1}}$, then $\max _{x \in\left[0, L_{\sigma}^{+}\right]}\left(g_{\mathrm{b}} \mid S_{b}\left(t_{1}\right)=y\right)(x)=\left(\mathrm{g}_{\mathrm{b}} \mid S_{b}\left(t_{1}\right)=y\right)(0)$.

(b) Assume $0<z_{1}^{2}<1$ and $L_{\sigma}^{+}>2 \sqrt{c_{1} c_{2}\left(z_{1}^{-2}-1\right)}$. If $\frac{2 \sqrt{c_{1} c_{2}}}{z_{1}} \leq L_{\sigma}^{+} \leq y$, then

$$
\begin{aligned}
& \max _{x \in\left[0, L_{\sigma}^{+}\right]}\left(\mathrm{g}_{\mathrm{b}} \mid S_{b}\left(t_{1}\right)=y\right)(x)=\left(\mathrm{g}_{\mathrm{b}} \mid S_{b}\left(t_{1}\right)=y\right)\left(x_{b, \mathrm{C}}^{*}\right) . \text { If } 0<L_{\sigma}^{+}<\frac{2 \sqrt{c_{1} c_{2}}}{z_{1}}, \text { then } \\
& \max _{x \in\left[0, L_{\sigma}^{+}\right]}\left(\mathrm{g}_{\mathrm{b}} \mid S_{b}\left(t_{1}\right)=y\right)(x)=\left(\mathrm{g}_{\mathrm{b}} \mid S_{b}\left(t_{1}\right)=y\right)(0) .
\end{aligned}
$$

Proof. See Appendix B.

\section{Simulation Results}

Geometric Brownian motion is a common and popular financial model. Its tractable feature allows it to have wide applications in financial practice. However, its positive trajectory prevents it modeling the occurrence of bankruptcy. This crucial risk factor should be considered, especially in a severe economic situation such as that caused by the impacts of COVID-19. Hence, in this section, we discuss the influence of omitting bankruptcy risk when setting the operations of limit orders via sensitivity analysis.

To discuss the influence of bankruptcy risk via sensitive analysis, we set three models: the higher bankruptcy risk model with $c_{1}=5.0$ and $c_{2}=1.0$, the lower bankruptcy risk model with $c_{1}=4.001$ and $c_{2}=0.001$, and the no bankruptcy risk model with $c_{1}=4$ and $c_{2}=0$. Note that $c_{2}$ is a crucial parameter in model (1) to illustrate bankruptcy risk. If the value of $c_{2}$ is higher, then model (1) has higher bankruptcy risk. The no bankruptcy risk model with $c_{2}=0$ is geometric Brownian motion. We set the parameters $a^{2}=0.008$ and $b=0.002$ or -0.002 for a stock price with an upward or downward trend, respectively. We set the total sum of taxes and trading fees as proportion to the stock price with the constant rate $\sigma=0.4425 \%$. We assume that an investor enters a position with the stock price $S_{b}\left(t_{1}\right)=4$ and then the cost (or income) of her/his position is defined as $L=4 \times(1+\sigma)=4.0177$ (or $L=4 \times(1-\sigma)=3.9823$ ) for the long (or short) position. The interest rate is set as $r=1 \%$. We use the fmincon function in Matlab to compute the optimal limit price of a sell limit order. The optimal limit price of a buy limit order is obtained directly via Theorem 4 . The results including the optimal limit prices and their optimal expected discounted profits are listed in Tables 1 and 2 for the buy and sell limit orders, respectively.

Table 1. The optimal limit prices and their optimal expected discounted profits of the sell limit orders with $a^{2}=0.008, b=0.002$ or $-0.002, r=0.01$, and $L=4.0177$.

\begin{tabular}{ccccc}
\hline & & $\begin{array}{c}\text { Higher } \\
\text { Bankruptcy } \\
\text { Risk }\end{array}$ & $\begin{array}{c}\text { Lower } \\
\text { Bankruptcy } \\
\text { Risk }\end{array}$ & $\begin{array}{c}\text { No Bankruptcy } \\
\text { Risk }\end{array}$ \\
\hline$b=0.002$ & $\begin{array}{c}\text { Optimal limit price } \\
\text { Optimal expected } \\
\text { discounted profit }\end{array}$ & 16.1694 & 15.5430 & 15.5401 \\
\hline$b=-0.002$ & $\begin{array}{c}\text { Optimal limit price } \\
\text { Optimal expected } \\
\text { discounted profit }\end{array}$ & 7.3460 & 1.8217 & 1.8315 \\
\hline
\end{tabular}


Table 2. The optimal limit prices and their optimal expected discounted profits of the buy limit orders with $a^{2}=0.008, b=0.002$ or $-0.002, r=0.01$, and $L=3.9823$.

\begin{tabular}{ccccc}
\hline & & $\begin{array}{c}\text { Higher } \\
\text { Bankruptcy } \\
\text { Risk }\end{array}$ & $\begin{array}{c}\text { Lower } \\
\text { Bankruptcy } \\
\text { Risk }\end{array}$ & $\begin{array}{c}\text { No Bankruptcy } \\
\text { Risk }\end{array}$ \\
\hline$b=0.002$ & $\begin{array}{c}\text { Optimal limit price } \\
\text { Optimal expected } \\
\text { discounted profit }\end{array}$ & 1.4282 & 2.5631 & 2.5740 \\
\hline$b=-0.002$ & Optimal limit price & 0.6221 & 0.6218 & 0.6178 \\
& $\begin{array}{l}\text { Optimal expected } \\
\text { discounted profit }\end{array}$ & 1.3654 & 0.7975 & 2.2782 \\
\hline
\end{tabular}

Intuitively, omitting the occurrence of bankruptcy could result in a higher optimal limit price. In Tables 1 and 2, all of the cases satisfy this expectation except the case of the sell limit order with the stock price having an upward trend. It is attributed to the structure of model (1) in which the effect of the first term is higher and the effect of the second term is lower when the price is higher. In addition, for the sell limit order with a stock price with an upward trend, the higher value of $c_{1}$ could enhance the effect of the first term in model (1) such that the optimal limit prices of the higher and lower bankruptcy risk models are more than that of the no bankruptcy risk model. However, their optimal expected discounted profits of sell limit orders are still less than that of the no bankruptcy risk model regardless of whether the stock price is experiencing an upward or downward trend.

We next discuss the results of the sell limit order with a stock price with an upward trend. From Table 1, the optimal prices are close in the three models when the stock price has an upward trend. This situation is attributed to the property that a higher $\left\{S_{b}(t)\right\}$ accompanies the lower effect of the minus term of $\left\{S_{b}(t)\right\}$ and then causes lower bankruptcy risk. Although the optimal limit price of the higher bankruptcy model with an upward trend is more than that of the no bankruptcy risk model, and its optimal profits are still less than those of the no bankruptcy risk model. Hence, the model that considers the bankruptcy risk indeed results in lower optimal profits when trading using a sell limit order. However, the results of the three models are close when the stock price has an upward trend.

When the stock price has a downward tendency, omitting the occurrence of bankruptcy results in a considerable difference between the higher bankruptcy risk model and the no bankruptcy risk model for the sell limit order. From Table 1, the optimal price of the higher bankruptcy risk model is $17.68 \%$ less than that of the no bankruptcy model and the optimal expected discounted profits of the higher bankruptcy risk model are $78.46 \%$ less than those of the no bankruptcy risk model. This table reveals that the omission of bankruptcy risk has a considerable influence on a sell limit order when the stock price has a downward trend. Owing to the lower optimal limit price of the higher bankruptcy risk model, setting the optimal limit price considering bankruptcy risk can provide more conservative operations for an investor with a long position when the stock price has a downward trend.

Next, we discuss the results of the buy limit orders. Since bankruptcy is considered in our model, the behavior of $\left\{S_{b}(t)\right\}$ should be lower than that of the no bankruptcy risk model. Hence, for a buy limit order, we can anticipate that the optimal limit prices of the higher and lower bankruptcy risk models should be less than that of the no bankruptcy risk model. Table 2 truly reflects this situation. Regardless of whether the stock price experiences an upward or downward trend, the optimal limit price of the higher bankruptcy risk model is less than that of the no bankruptcy risk model, and the optimal expected discounted profits of the higher bankruptcy risk models are over $65 \%$ more than those of the no bankruptcy risk model. For the stock price with a downward trend, it is noted that the optimal buy limit price of the higher bankruptcy risk model is $72.69 \%$ less than that of the no bankruptcy risk model. Therefore, under a severe economic crisis such as that caused by 
the COVID-19 pandemic, an investor with a short position can adopt the model including bankruptcy risk to set the limit price of a buy limit order in order to obtain better expected returns, which corresponds to the actual conditions.

Summarizing the results in Tables 1 and 2, the optimal limit prices and optimal expected discounted profits of the lower bankruptcy risk model are close to those of the no bankruptcy risk model. Hence, setting the optimal limit price based on the no bankruptcy model is indeed a good method to approximate the stock price with lower bankruptcy risk. However, especially for the results of the stock price with a downward trend, there are considerable gaps between the higher bankruptcy risk model and the no bankruptcy risk model. Consequently, when a firm is exposed to higher bankruptcy risk such as that due to the impacts of the COVID-19 pandemic and has a downward trend in its stock price, bankruptcy risk should be integrated into the stock price model so that an investor sets a reasonable optimal limit price for a limit order.

\section{Conclusions}

The omission of the bankruptcy risk in geometric Brownian motion could cause higher estimations of stock prices and then greatly influence the setting of trading strategies. To make geometric Brownian motion integrate bankruptcy risk, we consider an extended model of geometric Brownian motion and study its optimal limit price problem. We provide explicit expressions for the expected discounted profit functions and establish the optimal limit prices based on the profit criterion. We verify the existence of the optimal sell limit price and present the explicit form for the optimal buy limit price.

We conduct sensitivity analysis to assess the influence of the omission of bankruptcy risk. The numerical results reveal that omitting bankruptcy in the model indeed results in a higher optimal limit price except in the case of the sell limit order with the stock price having an upward trend. This exception is attributed to the higher value of $c_{1}$ enhancing the effect of the first term in model (1) and the higher stock price reducing the effect of the second term in model (1).

For the sell limit order with a stock price with an upward trend, both the optimal limit price in the three models, the higher bankruptcy risk model, the lower bankruptcy risk model and the no bankruptcy risk model, are similar since a higher value of model (1) results in low bankruptcy risk, i.e., a lower effect of the minus term in model (1).

For the limit order with stock price having a downward trend, there are considerable differences between the higher bankruptcy risk model and the no bankruptcy risk model for the optimal limit price. Our model can provide more conscientious operations for the sell limit order and achieve better expected discounted profits for the buy limit order when the stock price has a downward trend. Hence, under a severe economic crisis such as that caused by the impacts of the COVID-19 pandemic, integrating bankruptcy risk into the underlying asset price model not only provides the investor with a long position a cautious limit price setting for executing a sell limit order, but it also results in higher profits for an investor with a short position to execute a buy limit order.

A possible direction of future research is to extend model (1) with fractional Brownian motion. Recently, the fractional Brownian motion has fruitful applications in finance such as financial modelling, option pricing, optimal portfolio selection and high frequency trading, see Guasoni et al. [13], Guasoni et al. [14], Kř́ž and Szała [15] and Rostek and Schöbel [16] for more details. Another interesting issue of future research is to explore other real problems in finance market based on model (1) and test the efficiency of model (1) via analyzing the real financial data.

Author Contributions: Conceptualization, Y.-S.H. and C.-H.W.; methodology and validation, Y.-S.H., P.-C.C. and C.-H.W.; software, P.-C.C. and C.-H.W.; investigation, C.-H.W.; data curation, P.-C.C. and C.-H.W.; writing-original draft preparation, Y.-S.H. and C.-H.W.; and writing-review and editing, Y.-S.H., P.-C.C. and C.-H.W. All authors have read and agreed to the published version of the manuscript. 
Funding: This research received no external funding.

Institutional Review Board Statement: Not applicable.

Informed Consent Statement: Not applicable.

Data Availability Statement: Data is contained within the article.

Acknowledgments: We are grateful to the editor and three anonymous referees for their helpful and constructive suggestions and comments.

Conflicts of Interest: The authors declare no conflict of interest.

\section{Appendix A. The Proof of Theorem 3}

In this appendix, we verify Theorem 3. Let $w=x+\sqrt{x^{2}+4 c_{1} c_{2}}$ and $x=\frac{1}{2} w-$ $2 c_{1} c_{2} w^{-1}$. We can rewrite $\left(\mathrm{g}_{\mathrm{s}} \mid S_{b}\left(t_{1}\right)=y\right)(x)$ as:

$$
\left(\mathrm{g}_{\mathrm{s}} \mid S_{b}\left(t_{1}\right)=y\right)(x)=(1-\sigma)\left\{\frac{h_{1}(w) K_{1, y} w^{\frac{\mu}{a}+z}-L_{\sigma}^{-} e^{\mu D_{1}(0, y)} h_{2}(w)}{K_{3} w^{2 z+1}-\left(K_{3}\right)^{-1} w}\right\},
$$

where $z=\frac{\mu_{r}}{a}, h_{1}(w)=w^{2}-2 L_{\sigma}^{-} w-4 c_{1} c_{2}, h_{2}(w)=K_{2, y} w^{2 z+1}-\left(K_{2, y}\right)^{-1} w$,

$$
\begin{gathered}
K_{1, y}=\left(y+\sqrt{y^{2}+4 c_{1} c_{2}}\right)^{-\frac{\mu}{a}} \sinh \left(\mu_{r} D_{1}(y, 0)\right), \\
K_{2, y}=\left(y+\sqrt{y^{2}+4 c_{1} c_{2}}\right)^{-z} \text { and } K_{3}=\left(4 c_{1} c_{2}\right)^{-\frac{z}{2}} \text {. Obviously, we have: } \\
\lim _{x \rightarrow \infty}\left(g_{s} \mid S_{b}\left(t_{1}\right)=y\right)(x)=-(1-\sigma) L_{\sigma}^{-} e^{\mu D_{1}(0, y)}\left(\frac{K_{2, y}}{K_{3}}\right)=-L e^{\mu D_{1}(0, y)}\left(\frac{\sqrt{4 c_{1} c_{2}}}{y+\sqrt{y^{2}+4 c_{1} c_{2}}}\right)^{z} .
\end{gathered}
$$

Next, we will clarify that:

$$
\left(\mathrm{g}_{\mathrm{s}} \mid S_{b}\left(t_{1}\right)=y\right)\left(L_{\sigma}^{-}\right)=-L e^{\mu D_{1}(0, y)} \frac{\sinh \left(\mu_{r} D_{1}\left(L_{\sigma}^{-}, y\right)\right)}{\sinh \left(\mu_{r} D_{1}\left(L_{\sigma}^{-}, 0\right)\right)}>-L e^{\mu D_{1}(0, y)}\left(\frac{K_{2, y}}{K_{3}}\right) .
$$

Note that $\sinh \left(\mu_{r} D_{1}(L, y)\right)>0$ and $\sinh \left(\mu_{r} D_{1}(L, 0)\right)>0$ based on $D\left(L_{\sigma}^{-}\right)-D(0)>$ $D\left(L_{\sigma}^{-}\right)-D(y) \geq 0$. First, we claim that:

$$
\frac{\sinh \left(\mu_{r} D_{1}\left(L_{\sigma}^{-}, y\right)\right)}{\sinh \left(\mu_{r} D_{1}\left(L_{\sigma}^{-}, 0\right)\right)}<\frac{K_{2, y}}{K_{3}} .
$$

It is clear that

$$
\frac{\sinh \left(\mu_{r} D_{1}\left(L_{\sigma}^{-}, y\right)\right)}{\sinh \left(\mu_{r} D_{1}\left(L_{\sigma}^{-}, 0\right)\right)}-\frac{K_{2, y}}{K_{3}}=\left(\frac{\sqrt{4 c_{1} c_{2}}}{L_{\sigma}^{-}+\sqrt{\left(L_{\sigma}^{-}\right)^{2}+4 c_{1} c_{2}}}\right)^{z} \frac{\sinh \left(\mu_{r} D_{1}(0, y)\right)}{\sinh \left(\mu_{r} D_{1}\left(L_{\sigma}^{-}, 0\right)\right)} .
$$

Since $D_{1}(0, y)<0$ and $D_{1}\left(L_{\sigma}^{-}, 0\right)>0$, we obtain $\sinh \left(\mu_{r} D_{1}(0, y)\right)<0$ and $\sinh \left(\mu_{r} D_{1}\left(L_{\sigma}^{-}, 0\right)\right)>0$. Then, we verify that:

$$
\frac{\sinh \left(\mu_{r} D_{1}\left(L_{\sigma}^{-}, y\right)\right)}{\sinh \left(\mu_{r} D_{1}\left(L_{\sigma}^{-}, 0\right)\right)}<\frac{K_{2, y}}{K_{3}} .
$$

Hence, we have:

$$
\left(\mathrm{g}_{\mathrm{s}} \mid S_{b}\left(t_{1}\right)=y\right)\left(L_{\sigma}^{-}\right)>-L e^{\mu D_{1}(0, y)}\left(\frac{K_{2, y}}{K_{3}}\right) .
$$


We choose a sufficiently small $\epsilon>0$ small such that:

$$
\left(\mathrm{g}_{\mathrm{s}} \mid S_{b}\left(t_{1}\right)=y\right)\left(L_{\sigma}^{-}\right)>\epsilon-L e^{\mu D_{1}(0, y)}\left(\frac{K_{2, y}}{K_{3}}\right) .
$$

Since $\lim _{x \rightarrow \infty}\left(\mathrm{g}_{\mathrm{s}} \mid S_{b}\left(t_{1}\right)=y\right)(x)=-L e^{\mu D_{1}(0, y)}\left(\frac{K_{2, y}}{K_{3}}\right)$, there is a $\delta>0$ such that:

$$
-\epsilon-L e^{\mu D_{1}(0, y)}\left(\frac{K_{2, y}}{K_{3}}\right)<\left(\mathrm{g}_{\mathrm{s}} \mid S_{b}\left(t_{1}\right)=y\right)(x)<\epsilon-L e^{\mu D_{1}(0, y)}\left(\frac{K_{2, y}}{K_{3}}\right),
$$

for $x \in(\delta, \infty)$. We obtain $\max _{x \in\left[L_{\sigma}^{-}, \infty\right)}\left(\mathrm{g}_{\mathrm{s}} \mid S_{b}\left(t_{1}\right)=y\right)(x)=\max _{x \in\left[L_{\sigma}^{-}, \delta\right]}\left(\mathrm{g}_{\mathrm{s}} \mid S_{b}\left(t_{1}\right)=y\right)(x)$. Since $\left(\mathrm{g}_{\mathrm{s}} \mid S_{b}\left(t_{1}\right)=y\right)(x)$ is continuous on $\left[L_{\sigma}^{-}, \delta\right]$, we verify that $\left(\mathrm{g}_{\mathrm{s}} \mid S_{b}\left(t_{1}\right)=y\right)(x)$ attains the maximum value on $\left[L_{\sigma}^{-}, \infty\right)$ via the extreme value theorem.

\section{Appendix B. The Proof of Theorem 4}

We provide the proof of Theorem 4 in this appendix. The derivative of $\left(\mathrm{g}_{\mathfrak{b}} \mid S_{b}\left(t_{1}\right)=y\right)(x)$ follows:

$$
\left(\mathrm{g}_{\mathrm{b}} \mid S_{b}\left(t_{1}\right)=y\right)^{\prime}(x)=(1+\sigma)\left(\frac{x+\sqrt{x^{2}+4 c_{1} c_{2}}}{y+\sqrt{y^{2}+4 c_{1} c_{2}}}\right)^{z_{1}}\left[\frac{z_{1}\left(L_{\sigma}^{+}-x\right)-\sqrt{x^{2}+4 c_{1} c_{2}}}{\sqrt{x^{2}+4 c_{1} c_{2}}}\right] .
$$

Based on the similar discussion in the proof of Theorem 2, we obtain that:

$$
x_{b, C}^{*}=\frac{L_{\sigma}^{+} z_{1}^{2}-\sqrt{z_{1}^{2}\left(L_{\sigma}^{+}\right)^{2}+4 c_{1} c_{2}\left(z_{1}^{2}-1\right)}}{z_{1}^{2}-1}
$$

is the only candidate for the critical number on $\left[0, L_{\sigma}^{+}\right]$. Based on the same discussion in the proof of Theorem 2, we can prove (a). Assume $z_{1}^{2}>1$. If $\frac{2 \sqrt{c_{1} c_{2}}}{z_{1}} \leq L_{\sigma}^{+}$, then $0 \leq x_{b, C}^{*} \leq L_{\sigma}^{+}$. Since:

$$
\left(\mathrm{g}_{\mathrm{b}} \mid S_{b}\left(t_{1}\right)=y\right) \prime(x)\left\{\begin{array}{l}
\geq 0, \quad \text { if } x \in\left[0, x_{b, c}^{*}\right], \\
\leq 0, \quad \text { if } x \in\left[x_{b, C}^{*}, L_{\sigma}^{+}\right],
\end{array}\right.
$$

we have $\max _{x \in\left[0, L_{\sigma}^{+}\right]}\left(g_{\mathfrak{b}} \mid S_{b}\left(t_{1}\right)=y\right)(x)=\left(\mathrm{g}_{\mathfrak{b}} \mid S_{b}\left(t_{1}\right)=y\right)\left(x_{b, C}^{*}\right)$. If $0<L_{\sigma}^{+}<\frac{2 \sqrt{c_{1} c_{2}}}{z_{1}}$, then $x_{b, C}^{*}<0$ and $\max _{x \in\left[0, L_{L}^{+}\right]}\left(\mathrm{g}_{\mathrm{b}} \mid S_{b}\left(t_{1}\right)=y\right)(x)=\left(\mathrm{g}_{\mathrm{b}} \mid S_{b}\left(t_{1}\right)=y\right)(0)$. The similar arguments applied in (a) can also give the conclusion in (b).

\section{References}

1. McDonald, R.; Siegel, D. The value of waiting time to invest. Q. J. Econ. 1986, 101, 707-727. [CrossRef]

2. Shiryaev, A.; Xu, Z.; Zhou, X.Y. Thou shalt buy and hold. Quant. Finan. 2008, 8, 765-776. [CrossRef]

3. Dai, M.; Yang, Z.; Zhong, Y. Optimal stock selling based on the global maximum. SIAM J. Control Optim. 2012, 50, 1804-1822. [CrossRef]

4. Handa, P.; Schwartz, R.A. Limit order trading. J. Financ. 1996, 51, 1835-1861. [CrossRef]

5. Lo, A.W.; Mackinlay, A.C.; Zhang, J. Econometric models of limit-order executions. J. Financ. Econ. 2002, 65, 31-71. [CrossRef]

6. Agliardi, R. Modeling uncertainty in limit order execution. Commun. Nonlinear Sci. Numer. Simulat. 2016, 31, 143-150. [CrossRef]

7. Agliardi, R.; Gencay, R. Optimal trading strategies with limit orders. Int. J. Theor. Appl. Financ. 2017, 20, 1750005. [CrossRef]

8. Goodell, J.W. COVID-19 and finance: Agendas for future research. Financ. Res. Lett. 2020, 35, 101512. [CrossRef]

9. Baldwin, R.; Weder di Mauro, B. (Eds.) Economics in the Time of COVID-19; CEPR Press: London, UK, 2020.

10. Zhang, D.; Hua, M.; Ji, Q. Financial markets under the global pandemic of COVID-19. Financ. Res. Lett. 2020, 36, 101528. [CrossRef] [PubMed]

11. Hsu, Y.S.; Wu, C.H. Extended Black and Scholes model under bankruptcy risk. J. Math. Anal. Appl. 2020, 482, 123564. [CrossRef]

12. Karatzas, I.; Shreve, S.E. Brownian Motion and Stochastic Calculus, 2nd ed.; Springer: New York, NY, USA, 1991.

13. Guasoni, P.; Mishura, Y.; Rásonyi, M. High-frequency trading with fractional Brownian motion. Financ. Stoch. 2020. [CrossRef]

14. Guasoni, P.; Nika, Z.; Rásonyi, M. Trading fractional Brownian motion. SIAM J. Financ. Math. 2019, 10, 769-789. [CrossRef] 
15. Kř́ǐz, P.; Szała, L. Least-squares estimators of drift parameter for discretely observed fractional Ornstein-Uhlenbeck processes. Mathematics 2020, 8, 716. [CrossRef]

16. Rostek, S.; Schöbel, R. A note on the use of fractional Brownian motion for financial modeling. Econ. Model. 2013, $30,30-35$. [CrossRef] 